\title{
Ergonomic tridimensional analysis: critical ergonomic factors identification in a commercial environmental
}

\author{
Loureiro, I.F: ${ }^{\mathrm{a},}{ }^{*}$, Leão, C.P. ${ }^{\mathrm{a}}$ and Arezes, P.M. ${ }^{\mathrm{a}}$ \\ a Department of Production and Systems Engineering, University of Minho, Campus de Azurém, Guimarães, \\ Portugal
}

\begin{abstract}
The Ergonomic tridimensional Analysis (ETdA) was developed to be used as an auxiliary tool on the ergonomic intervention. It was specially designed for commercial areas with free circulation of people. Due to that, the client, the third ETdA dimension, becomes an important element and their opinions relevant in the ergonomic analysis. The remains two ETdA dimensions, professionals and analyst, play an identical role as in the traditional occupational ergonomic analysis. For each of these dimensions, specific observation tools were assembled: an ETdA questionnaire, an evaluation form and a checklist for direct and indirect observations. The variables that allow the ETdA operability are identified as Ergonomic Factors (EFs). A case study is presented identifying, for each dimension, the critical EFs. This will allow the design of the weighting table, where the influence of each dimensions results is measured, stressing the important role of clients on ergonomic issues.
\end{abstract}

Keywords: commercial workplace environment, ergonomic factors, ergonomic tridimensional analysis, weighting table

*Corresponding author: ID25001@alunos.uminho.pt 


\section{Introduction}

The economic market diversification allows the differentiation of a greater number of ergonomic contexts. The appearance of large Commercial Areas with Free Circulation of People (CAFCP) represents a social-technical system that must be considered from an occupational and usability point of view [5, 13]. In fact, in these areas, the professionals' activities are related with clients or consumers service or products sales (common areas). This situation provides a human interaction. From the management point of view, the ongoing concern with the client requires a continuous improvement in several organizational key areas such as quality, occupational safety and health, natural environment protection, and cost of products and services [5]. As a result of this market customization, the organizational adjustments taking place on the common areas must also benefit the ergonomic context of the professionals. Following these thoughts, to optimize the performance of the overall system (economical goal), the organizations' strategies and goals must also consider the human wellbeing (social goal) [3, 9]. In the common areas, the human wellbeing is related with its users, both the clients and professionals.

Ergonomic Tri-dimensional Analysis (ETdA) is a new approaching developed specifically for ergonomic assessments and redesign of Common Areas with Free Circulation of People (CAFCP). It is a continuous model that assists the ergonomist (Analyst) in his/her ergonomic analysis leading to the establishment of priorities list. The analysis is multidimensional since it considers all the organizational participants, clients and professionals, and the analyst. The variables analyzed with this model are called Ergonomic Factors (EFs) and allow the ETdA operability. They can be divided into two major groups: intrinsic (individual: work postures, general physical activity, communication/interrelation and attentiveness) and extrinsic (environmental: noise, lighting, thermal environment and risk accident or occupational: professional training quality, job content, decision making, restrictiveness) [10]. The set of $14 \mathrm{EFs}$ is flexible, and it can be chosen according to the area under analysis. The diagnosis of the studied conditions and identification of the critical EFs and consequent adjustments represent the ergonomic intervention. Observation tools were assembling to each one of the three dimensions, to know: a questionnaire, an evaluation sheet and direct and indirect observation (checklist) for the clients, professionals and analyst dimensions, respectively.

The current study was carried out within a large departmental store characterized by common areas where professionals and clients interact and can be exposed to the same ergonomic risk factors [14]. The purpose of this work was to use the ETdA model to identify the critical EFs according to the severity of the identified situation and to understand all the mechanisms that regulate the three ETdA dimensions relationships: Analyst/Professionals (AP), Analyst/Clients (AC), Clients/Professionals (CP) and Management/Clients/Analyst (MCA)) [10]. In these large common areas, since different ergonomic contexts can be identified, analyst and professionals dimensions analysis was done by section and according to the qualified activity. The ETdA questionnaire was properly adapted and applied. In this stage, the communication channel between store manager and analyst had an important role contributing to some environment quality issues, i.e., utility, functionality, environmental adjustment, aesthetics, prestige, usability and pleasure [4, 15]. The three categories of dimensions answers are assorted in an ascending order according to the seriousness of the situation. A methodology for negatives categories' analysis was developed by using a standardized residuals contribution. This procedure increases the meaningfulness of the obtained results. It is authors' believe that it is easier to propose changes when the client has similar opinion to the analyst and/or the professional. ETdA model follows the emerging challenges in the ergonomics domains by increasing the population awareness regarding ergonomics, allowing the participation of the entire organization in critical situations' identification and proposals of intervention $[2,10]$.

\section{Methodology}

The Ergonomic analysis was conducted using the ETdA methodology created explicitly for CAFCP. A case study based in a Wholesale retail store ergonomic analysis was performed. This commercial area comprises a large open space where a wide variety of products are displayed; identifying different ergonomic contexts with specific professionals' activities. This case study aims at identifying the critical EFs and the three ETdA dimensions' relationships understandings. 


\subsection{ETdA planning}

In order to encourage the participation of the entire organization in the ergonomic analysis, ETdA planning was carefully defined with the store manager. The analyst oncoming to the store manager create co-responsibility in the ergonomic intervention decision making. The modus operandi of the assessment is dependent on the ergonomics multiplicity contexts identified on the commercial area under study. The common area was divided in sectors according to the professional activity identified. The observation tool for the professional dimension was delivered by sector thus, making it possible to obtain a global and by professional activity profile. The ETdA questionnaire was suitably adapted and applied randomly during three months. Field force available in the store was used contributing to the success of this particularly task. In the Analyst dimension, direct and indirect observations (checklist) were used to classify the ergonomic contexts previously identified.

This case study aims at identifying the critical EFs and the three ETdA dimensions' relationships understandings.

\subsection{Data collection and analysis}

This section is related to the data collection and the tri-dimensional results' analysis of the studied EFs (see Introduction). In ETdA the observation tools are: evaluation forms and direct and indirect observation to professionals and analyst dimensions, respectively. A questionnaire was developed for the dimension of clients. It is a well-documented device that exists to help the evaluation of task problems in CAFCP. This tool allows a client's easy approach and respectively characterization and the identification of critical ergonomic factors in the common areas. The questionnaire was pre-tested to be used in the survey [8] and the results of validation (sensibility, validity and reliability) contributed for its improvement $[6,11]$.

Through a three level analysis, the results of the observation tools were studied. This analysis includes: (1) a descriptive analysis to allow the characterization and study of the different answers profile in clients and professional dimensions; (2) a correlation analysis between the different answer categories and the results of stage 1 ; (3) a multivariate analysis (inter and intra dimension analysis) of the ergonomic factors [12]. The observation tool for the clients' dimension, called ETdA questionnaire, also allows to gather some knowledge about the socio demographic characterization of the population, in particular of the characterization of the population of clients (clients dimension profile). A pre-processing of the obtained data must be done in order to simplify and summarize the dimensions results, leading to the three dimensions weighting table construction, which represents the ergonomic intervention proposals [7]. These tables support the analyst final task: real perception of the ergonomic situation and elaboration of the priority list of changes to be implemented according to the critical the identification of the critical gravity EF. The three dimensions categories' answers are assorted in an ascending order according to the seriousness of the situation. In ETdA terminology they are divided in negative or positive categories; for instance, considering the lightning quality EFs, the categories "very bad", "bad" are negative and the "good" and "very good" categories are positive one. Even thought the negative categories can represent a lower answer' percentage, their existence should be considered relevant to the ergonomic analysis. In effect, they can highlight the se-verity of a particular situation, representing an indicator of the need for a supplementary ergonomic study. Given that, it is not possible to eliminate those categories from the analysis. A methodology for negatives categories' analysis was developed by using a standardized residuals contribution. This procedure increases the meaningfulness of the obtained results.

At the end, a dimension profile is obtained, and several correlations can be studied, leading to each dimension the identification of the critical ergonomic factors of the entire organization in critical situations' identification and proposals of intervention.

\section{Results}

\subsection{Dimensions profile}

From the total delivered questionnaires in the clients' dimension, the results reveal that 129 clients participated in the case study, yielding a $43 \%$ response rate. Clients' age ranged between 17 to 76 years old, with an average age of 49 . About $66.1 \%$ of them were male. It is interesting to note that in a five points scale (in an increasing order level of opinion), clients have considerable knowledge regarding to ergonomic issues and are regular clients (85\%) [5]. 
In professionals' dimension, and based on a total of 183 professionals, $58 \%$ reported a positive general answer classification. The thermal environment evaluation was the only ergonomic factor that reveals substantial differences.

\subsection{Critical Ergonomic Factors: identification}

In this case study, risk ergonomic factors were identified according to the highest negative categories' percentage reported in clients and professionals dimensions, which in this case was the thermal environment, noise and the adopted postures and movements and lifting.

Through an intra analysis of the clients and professionals dimensions', thermal environment evaluation results showed that $69 \%$ of the professionals and $35.8 \%$ of the clients answers were related with the negative categories. The sections with highest negative categories percentage, reported by clients and professionals are presented in table 1 . The inter analysis results shows that the hypothesis that tests if the distribution of the professionals and clients thermal evaluation is the same across the dimensions is rejected $(\mathrm{p}<.001)$, and they are not significantly related $(\chi 2(2)=0.801, p>.05)$. However, the biggest standardizing residual value is associated with the positive category in clients dimension and negative in professionals' dimension. This suggests that when clients think that the temperature level is "good", more professionals than expected consider the temperature as an ergonomic risk factor. Analyst has identified the Grocery, dairy and butchery sections, Fish and Freezing area, Fruits and vegetables as the most critical areas.

Considering the noise factor, $20.9 \%$ of the professionals considered the existence of a problem in the section where they develop their activity. Most of the answers were relate to the "front office" or "checkout". In the clients' dimension, $7.8 \%$ of them reported this situation. Even though the small obtained percentage, all the sections were reported. The inter analysis results shows that the noise factor evaluation distribution is significantly independent of the ETdA dimension $(\mathrm{p}>0.05)$ and they are not significantly related $(\chi 2(4)=7.794, p>0.05)$. Results show that clients' positive classification is related with negative professionals' evaluation. This factor seems to indicate that the time of exposure is important in the professional evaluation, i.e., the noise problem identified by professionals in a particularly sector does not seem to bother clients in their shops. Analyst had a positive evaluation of this ergonomic factor in all the analyzed sections.

The EFs for postures and movements and lifting tasks were related with clients and professional behaviour. Amongst the professionals' evaluation, $43.4 \%$ of them reported negative answers' categories in the EF postures and movements and $48.6 \%$ have the same classification for the other EF. In clients' dimension, $21.7 \%$ had difficulty to reach products on the shelves and $50.5 \%$ of them had difficulties to operate the shopping trolleys, in particular when they are fully loaded, i.e., in situation where the total weight can reach values of approximately $600 \mathrm{~kg}$. Client's difficulties to operate the shopping trolleys have a significant effect on the shopping trolley evaluation. Results show that clients' difficulties to operate the shopping trolleys are highly associated with the clients' shopping trolleys evaluation $(\chi 2$ (25) $=80.891, \mathrm{p}<0.001)$, and the existence of obstacles in the passage $(\chi 2(4)=37.162, p<0.001)$. This suggests that when clients think that the shopping trolley quality is "bad" more clients than expected have difficulties in operate them. The existence of obstacles seems to contribute to an inadequate posture and movements of the clients. The inter analysis shows that the distribution of the results for the EFs for postures and movements and lifting tasks is independent from the evaluation dimensions $(\mathrm{p}>.05)$.

Table 1

Sections with highest negative categories percentage for thermal environment evaluation

\begin{tabular}{|l|l|}
\hline Dimension & Sections \\
\hline \multirow{4}{*}{ Clients } & Grocery and Fish area \\
\cline { 2 - 2 } & Clients' reception area and Front office \\
\cline { 2 - 2 } & Freezing area \\
\cline { 2 - 2 } & Fruits and vegetables \\
\hline \multirow{4}{*}{ Professionals } & Grocery and Fish area \\
\cline { 2 - 2 } & Fruits and vegetables \\
\cline { 2 - 2 } & Beverage areas \\
\cline { 2 - 2 } & Office media \\
\cline { 2 - 2 } & Clients' reception area and Front office \\
\hline
\end{tabular}


Helping the Lifting EF classification decision in clients' and professionals' dimension, a rescaled to a three level scale was done. According to the seriousness of the situation three categories are proposed: "bad", "satisfactory" and "good". Full answer distribution is represented in Figure 1 and 2. Results show that most of clients' answers had positive classifications ("good" and "satisfactory") (Figure 1). Meaning that clients' considered this EF does not represent an ergonomic risk. In opposition, the answers' distribution in professionals' dimension shows a negative tendency. However, the "satisfactory" and "bad" categories distribution is similar (Figure 2). This result is in line with the ETdA conceptualization namely, the existence of categories showing the severity on a particular situation should be considered relevant and can represent an indicator of the need for a supplementary ergonomic study. This is why the final classification of the professionals dimension is lower than the clients' dimension.

\subsection{Critical Ergonomic Factors: Weighting table}

The critical ergonomic factors identified with the ETdA methodology; Thermal environment, Noise, Postures and movements and Lifting, accomplished by the three level analyses are shown in Table 2 . This table represents the weighting table matrix with a three points scale evaluation (1-negative 2-satisfactoy, and 3-positive).

Considering the thermal environment evaluation, there is an agreement between clients and analyst dimensions. The weighting results for the postures and movements EF are equal. Both professionals and

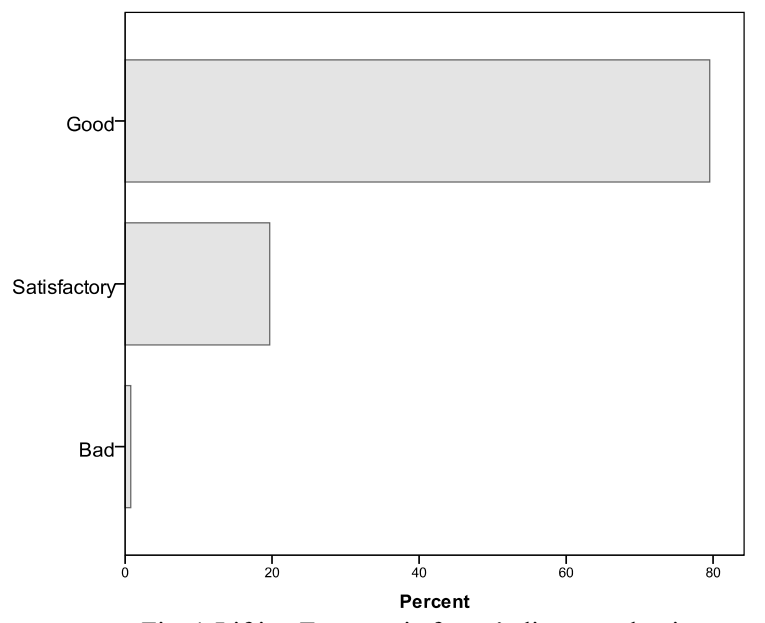

Fig. 1 Lifting Ergonomic factor' clients evaluation analyst dimensions have the same evaluation for the lifting EF. Different noise weighting results are obtained. This suggests that, although the measured values are within the limits allowed by law, issues such as the people well-being should also be considered. Therefore, the noise ergonomic factor should be studied well beyond the recommended values.

\section{General discussion}

The ETdA (Ergonomic Tri-dimensional Analysis) development follows the ergonomics future tendency since it allows the participation of the entire organization in the identification of critical situations and in making proposals of intervention. In order to identify the critical ergonomic factors in a commercial environmental, a case study was done in Wholesale retail store. Observation tools were applied to allow data collection in each dimension. According to the highest negative categories' percentage reported in clients and professionals dimensions, thermal environment, noise and the adopted postures, movements and lifting were the risk ergonomic factors identified. Through a data preprocessing and a three level analysis, the observation tools results were studied, allowing the definition of the clients dimension profile and to develop the weighting tables [14]. These tables can be defined as the simplification and summarization of the ETdA dimensions results, helping the Analyst in the decision making for ergonomic intervention.

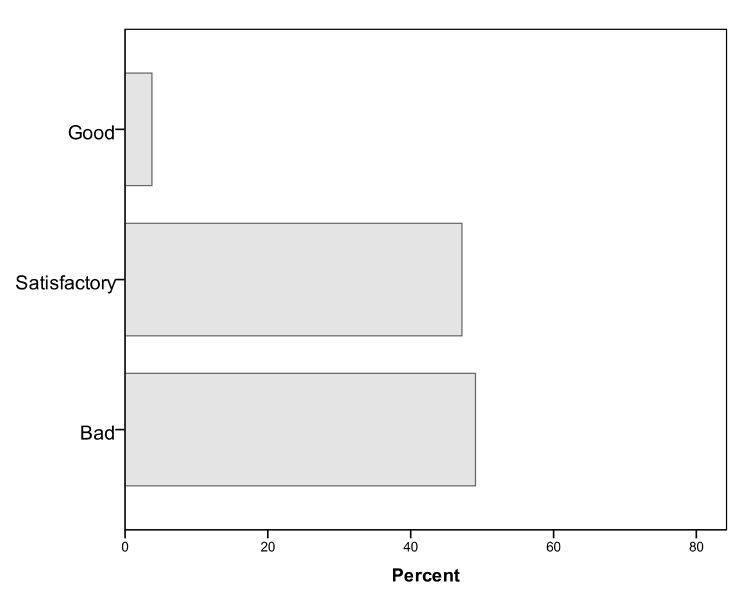

Fig. 2 Lifting Ergonomic factor' Professionals evaluation 
Table 2

ETdA matrix for Thermal, Noise, Postures and movements and Lifting EFs

\begin{tabular}{|l|c|c|c|}
\hline EF & $\begin{array}{c}\text { Professionals } \\
\text { dimension }\end{array}$ & $\begin{array}{c}\text { Clients } \\
\text { dimension }\end{array}$ & $\begin{array}{c}\text { Analyst } \\
\text { dimension }\end{array}$ \\
\hline Thermal & 1 & 2 & 2 \\
\hline Noise & 1 & 2 & 3 \\
\hline $\begin{array}{l}\text { Postures and } \\
\text { movements }\end{array}$ & 1 & 1 & 1 \\
\hline Lifting & 1 & $2-3$ & 1 \\
\hline
\end{tabular}

The obtained results also showed the importance of the clients' dimension in the Ergonomic analysis. The clients' evaluation can be used as an indicator of some situations that would otherwise not be priority for action. It can be useful to improve the analyst evaluation when there is agreement in the ratings (analyst, client, professional), or to be used as an advice-guider if it is inconsistent. It is authors' believe that any of these situations will have greater impact on the manager decision for the ergonomic intervention.

Finally, extending the ETdA issues will allow to understand and interpret the ergonomic context and to increase the Management/Client/Analyst relationship. Clients' organizational ongoing concern will reproduce adjustments, which will also benefit the professionals' ergonomic context, by facilitating the ergonomic intervention.

\section{References}

[1] J. Barling, E.K. Kelloway, and M.R. Frone, Handbook of work stress, SAGE, 2005.

[2] D.C. Caple, The IEA contribution to the transition of Ergonomics from research to practice, Applied Ergonomics, 41, (2010), 731-737.

[3] J. Dul and W.P. Neumann, Ergonomics contributions to company strategies, Applied Ergonomics, 40, (2009), 745-752.

[4] J.S. Dzissah, W. Karwowski, J. Rieger, and D. Stewart, Measurement of management efforts with respect to integration of quality, safety, and ergonomics issues in manufacturing industry, Human Factors and Ergonomics in Manufacturing \& Service Industries, 15, (2005), 213-232.

[5] B.H. Forcier, A.E. Walters, E.E. Brasher, J.W. Jones, Creating a safer working environment through psychological assessment: A review of a measure of safety consciousness, Journal of prevention and Intervention in the Community, 22: $1,(2001), 53-65$.

[6] A. Hedlund, M. Åteg, I.M. Andersson, and G. Rosén, Assessing motivation for work environment improvements: Internal consistency, reliability and factorial structure, Journal of Safety Research, 41, (2010), 145-151.
[7] J.R. Kettenring, Massive datasets, Wiley Interdisciplinary Reviews: Computational Statistics, 1, (2009), 25-32.

[8] H.M. Khalid; M.G. Helander, A framework for affective customer needs in product design, Theoretical Issues in Ergonomics Science, vol. 5,(2004), 27-42.

[9] K. Kogi, Participatory methods effective for ergonomic workplace improvement, Applied Ergonomics, 37, (2006), 547-554.

[10]I.F. Loureiro, C.P. Leão, e P.M. Arezes, Management of the Benefits on the Client's Involvement on Ergonomic Analysis, Communications in Computer and Information Science 92, Tenreiro de Magalhães et al.ed., Braga, 2010, 1-8.

[11]I.F. Loureiro, C.P. Leão, e P.M. Arezes, Ergonomic Tridimensional Analysis: exploratory analysis in clients' dimension observation tool, in: Selected Papers of the XVIII Congresso Anual da Sociedade Portuguesa de Estatística, Carlos Tenreiro ed., S. Pedro do Sul (Portugal), 1, 2010, 119 121.

[12]I.F. Loureiro, C.P. Leão, and P.M. Arezes, Clients, Professional and Analyst dimensions: intra and inter relationships analysis in the decision-making, in Proceedings 11 th annual meeting of the European Network for Business and Industrial Statistics (ENBIS), 2011, University of Coimbra (Portugal).

[13]L. Querelle and J.-F. Thibault, La pratique de l' intervention d'ergonomes consultants: une approche réflexive orientée par les outils.,@activités, 4, (2007), 149-159.

[14] Rajagopal, Coexistence and conflicts between shopping malls and street markets in growing cities: Analysis of shoppers' behavior, Journal of Retail and Leisure Property, 9, (2010), 277-301.

[15]C. Ziliani and S. Bellini, Retail Micro-Marketing Strategies and Competition, The International Review of Retail, Distribution and Consumer Research, 14, (2004), 7-18. 